\title{
Framework Conceitual para Formação de Grupos de Alunos utilizando Trilhas de Aprendizagem em um Ambiente Virtual de Aprendizagem
}

\author{
Ilmara Monteverde Martins Ramos ${ }^{1,2}$ David Brito Ramos ${ }^{1,2}$, Geovana de \\ Souza Amaral ${ }^{1}$, Bruno Freitas Gadelha ${ }^{1}$, Elaine Harada Teixeira de Oliveira ${ }^{1}$ \\ ${ }^{1}$ Instituto de Computação - Universidade Federal do Amazonas (UFAM) - Manaus - \\ AM - Brasil \\ ${ }^{2}$ Instituto Federal de Educação, Ciência e Tecnologia do Amazonas (IFAM) - Campus \\ Parintins - Parintins - AM - Brasil \\ \{ilmaramonteverde, davidness,gsamaral05\}@gmail.com, \\ brunodicomp.edu.br, elainedicomp.ufam.edu.br
}

\begin{abstract}
In this paper we propose a conceptual framework for groups formation of students in a Virtual Learning Environment (VLE). We have developed and validated a conceptual framework for group recommendation for collaborative activities, using student characterization based on learning paths (LPS) in a VLE. The approach we followed emphasizes group formation applying K-Means algorithm, using three similarity metrics: Euclidian, Manhattan and Cosine metrics. The framework validation was performed through the implementation of the M-Cluster tool. Real user interaction data with the tool is used in validation.
\end{abstract}

Resumo. Neste artigo é proposto um framework conceitual para formação de grupos de alunos em um Ambiente Virtual de Aprendizagem (AVA). Nós desenvolvemos e validamos um framework conceitual para recomendação de grupos para atividades colaborativas, utilizando a caracterização dos aprendizes baseada em trilhas de aprendizagem (TAs). A abordagem seguida dá ênfase à formação dos grupos aplicando o algoritmo K-Means, que é utilizado com três métricas de similaridade, que são as distâncias: Euclidiana, Manhattan e Cosseno. A validação do framework foi realizada por meio da implementação da ferramenta M-Cluster. Dados reais da interação de usuários com a ferramenta são utilizados na validação.

\section{Introdução}

Para que um grupo de indivíduos consiga realizar tarefas em conjunto, a colaboração é de fundamental importância, pois as discussões e o refinamento de ideias são fundamentais para o êxito nas tomadas de decisões do grupo [Natalli e Menezes 2012]. Diante disso, grupos são conjuntos pequenos de pessoas que se unem em prol de um objetivo [Santos, Castro e Castro 2007]. Formar grupos em um curso presencial ou a distância pode ser um processo não natural [Barkley, Major e Cross 2014].

Contudo, para que a aprendizagem colaborativa seja bem-sucedida, em qualquer modalidade é importante formar grupos que possam cumprir os objetivos da tarefa de forma satisfatória. Nesse sentido, busca-se melhorar as interações dos alunos em 
VII Congresso Brasileiro de Informática na Educação (CBIE 2018)

Anais do XXIX Simpósio Brasileiro de Informática na Educação (SBIE 2018)

atividades que requerem colaboração principalmente em Ambientes Virtuais de Aprendizagem (AVAs).

Nesse sentido, as Trilhas de Aprendizagem (TAs) podem ser consideradas como recursos para sugerir a formação de grupos de alunos. No contexto deste artigo, uma TA representa a trajetória percorrida pelo aluno durante a sua interação com o AVA sendo representada na forma de grafo [Ramos et.al. 2015]. O uso de TAs é promissor porque mostra os caminhos percorridos pelos alunos durante a sua interação com o AVA, o que possibilita sugerir grupos baseados nessas interações dado que formar grupos na modalidade EaD é realmente uma tarefa desafiadora para o docente.

No geral, nessa modalidade, os alunos só possuem $20 \%$ do contato presencial entre si e com o docente, dificultando estabelecer critérios para realizar a formação de grupos de alunos. Diante deste contexto, buscando contribuir com esta área, é apresentado neste artigo um framework conceitual para formação de grupos em atividades colaborativas, por meio dos dados extraídos das TAs propostas por [Ramos, et.al. 2017], para auxiliar o docente no processo de ensino-aprendizagem.

A abordagem seguida dá ênfase à formação dos grupos aplicando o algoritmo KMeans [MacQueen 1967], que é utilizado com três métricas de similaridade, que são as distâncias: Euclidiana, Manhattan e Cosseno, usando os atributos mais relevantes do aluno (média de acesso de vértices, quantidade, dispersão e variâncias das arestas padrão, avanço e retorno) obtidos dos dados extraídos das TAs.

Este artigo está estruturado da seguinte forma: na Seção 2 são discutidos os trabalhos relacionados. A Seção 3 descreve o processo metodológico para a criação do framework, enquanto a Seção 4, discute o framework conceitual proposto para formação de grupos de alunos. A ferramenta desenvolvida a partir da concepção do framework é apresentada na Seção 5. A validação inicial e os resultados estão na Seção 6, e por fim, a Seção 7 apresenta a conclusão e as sugestões para trabalhos futuros.

\section{Trabalhos relacionados}

Os trabalhos a seguir apresentam um panorama das diferentes formas de agrupamento de alunos em AVAs, como em [Jagadish 2014], que utilizada o KNN (K-Nearest Neighbor) para realizar agrupamento de alunos no Moodle. Os trabalhos de [Abnar, Orooji e Taghiyareh 2012] propõem o agrupamento com base em algoritmos genéticos e escala de Likert, tendo como diferencial a possibilidade de o professor poder escolher um conjunto de diferentes atributos e classificá-los com base em seu impacto sobre a formação de grupos. Já o trabalho de [Yathongchai et al. 2013] usa as técnicas de árvore de decisão, mineração de dados e índice de Hartigan para formar grupos de alunos no Moodle com base em suas notas individuais e comportamentos. A pesquisa de [Montazer e Rezaei 2013] aborda a criação de um método híbrido de agrupamento que consiste na junção dos métodos K-Means e Fuzzy C-Means [Bezdek 1981], este intitulado de Hybrid Clustering Method (HCM). Este método híbrido foi avaliado comparando com outros três métodos tradicionais: K-Means, Fuzzy C-Means e Evolutionary Fuzzy C-Means [Le 1995].

Como visto anteriormente, os trabalhos citados estão relacionados com esta proposta, sendo que as abordagens de [Jagadish 2014] realizam a formação de grupos de forma explícita, ou seja, os dados são coletados por meio de questionários, onde os alunos preenchem as informações que são usadas nos agrupamentos, já os trabalhos de 
VII Congresso Brasileiro de Informática na Educação (CBIE 2018)

Anais do XXIX Simpósio Brasileiro de Informática na Educação (SBIE 2018)

[Abnar, Orooji e Taghiyareh 2012], [Yathongchai et al. 2013], [Montazer e Rezaei 2013] formam os grupos de forma implícita, os dados são extraídos por meio de logs de acessos das plataformas utilizadas. A maioria dessas pesquisas usam o Moodle e técnicas de mineração de dados ou raciocínio baseado em casos. As fontes de dados usadas para caracterizar os perfis dos alunos nas duas primeiras pesquisas foram os questionários e das três últimas, os logs de acessos extraídos da plataforma Moodle. Logo, essa pesquisa difere das apresentadas por considerar as TAs para a formação dos grupos dos aprendizes. Além disso, nos trabalhos citados, a técnica de mineração de dados mais utilizada foi do K-Means com uma única métrica de similaridade, alcançando um único resultado. Já nesta abordagem, é utilizado o algoritmo K-Means com três métricas de distâncias: Euclidiana, Manhattan e Cosseno, onde cada métrica apresenta um resultado, oferecendo ao docente três possibilidades de recomendação de grupos de alunos de forma automática.

\section{Metodologia}

Foi realizada uma pesquisa bibliográfica por meio de uma Revisão Sistemática de Literatura (RSL) sobre recomendação de grupos para atividades colaborativas em ambientes virtuais. Tal revisão sistemática ocorreu por meio de consultas em bases de conhecimento científico, como: Scopus e IEEE, publicada em [Ramos et al. 2016], e uma pesquisa de campo, onde foram coletados dados de um AVA, durante dois semestres 2015/1 e 2016/1 da disciplina de Matemática Discreta. Os dados eram de 82 alunos de duas turmas já encerradas, de dois cursos à distância, uma de Ciência da Computação e a outra de Engenharia da Computação, Universidade Federal do Amazonas (UFAM), onde foram analisadas as interações registradas no banco de dados do AVA das atividades em grupo.

A partir das análises realizadas, os alunos são agrupados de acordo com o grau de similaridade, calculado usando-se os atributos extraídos das trilhas de aprendizagem. Esse agrupamento é representado de duas formas: gráfico de bolhas e lista descritiva. $\mathrm{O}$ gráfico de bolhas exibe os grupos em forma de bolha e dentro de cada bolha são apresentados os nomes dos alunos que pertencem ao mesmo grupo. Já a lista descritiva é uma lista não ordenada no qual aparecem uma identificação do grupo seguida de uma lista de nomes dos alunos que pertencem ao grupo. Em seguida, uma ferramenta foi desenvolvida para o Moodle na forma de plugin, para analisar o framework produzido. Os atributos foram analisados utilizando o algoritmo K-Means e três métricas de similaridade distintas para realizar o agrupamento e classificação dos grupos.

Assim, as formações de grupos de alunos foram analisadas utilizando técnicas de mineração de dados e aprendizagem de máquina, para a geração das sugestões de grupos de alunos. Posteriormente, foi realizada uma validação, com novos dados, em uma disciplina da UFAM, Matemática Discreta, de uma turma em andamento na modalidade presencial. Mais detalhes serão apresentados na seção 6 deste artigo.

\section{Arquitetura da Ferramenta}

Nesta seção apresentamos um framework conceitual denominado TARGAVA que tem como objetivo recomendar grupos de alunos para atividades colaborativas, utilizando a caracterização dos aprendizes baseadas nas TAs em um AVA, para auxiliar o professor no processo de ensino-aprendizagem. Foram realizadas coletas de dados do banco de dados do AVA Moodle, para fazer análises das TAs criadas por [Ramos et al. 2016], 
VII Congresso Brasileiro de Informática na Educação (CBIE 2018)

Anais do XXIX Simpósio Brasileiro de Informática na Educação (SBIE 2018)

buscando definir atributos para serem utilizados na concepção dos grupos de alunos. Esses atributos (mais detalhes na subseção 4.3) foram encontrados e validados, por meio de entrevistas com a professora especialista.

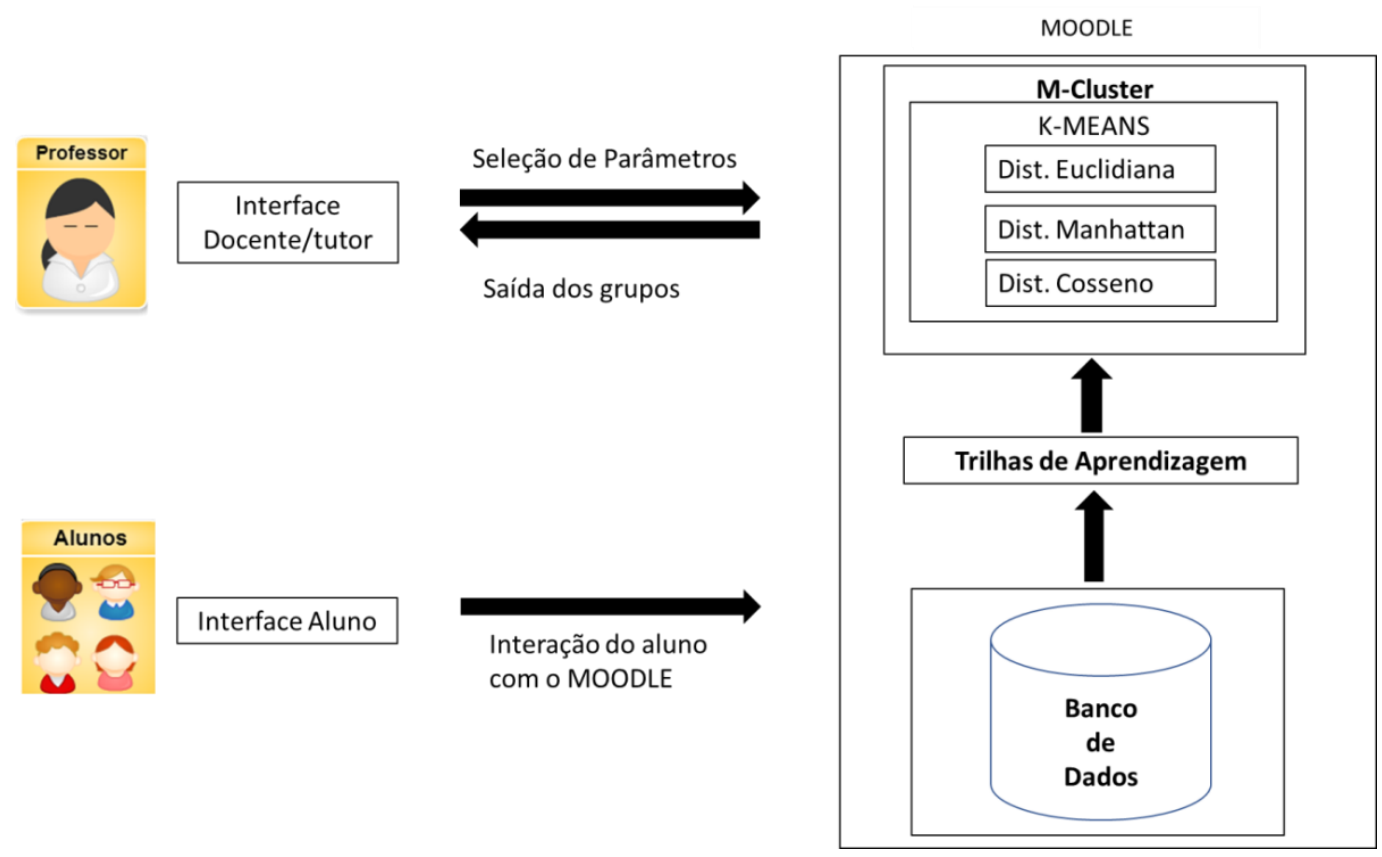

Figura 1: Visão Geral do Framework

Na Figura 1, acima, é apresentada uma visão geral do framework conceitual, este possui os seguintes componentes: a interface Aluno, Banco de dados, Trilhas de Aprendizagem, Formação de Grupos e Interface Docente/Tutor.

\subsection{Interface Aluno}

Nesta interface o aluno interage com o ambiente Moodle. Todas as suas ações/interações são guardadas no banco de dados. A partir disso, são extraídas suas preferências e interesses de forma automática, não necessitando de formulários para a coleta de informações, assim como sua interação durante o curso na plataforma virtual.

A seguir, serão descritos mais detalhes de como são armazenados esses dados e a estrutura preparada para realizar a organização dessas informações no banco de dados.

\subsection{Banco de dados}

Das informações armazenadas no banco de dados do Moodle, as mais relevantes para este trabalho são as relacionadas com as interações dos alunos durante o curso no AVA. A estrutura do banco de dados utilizada foi a definida por [Ramos et al. 2016]. Nesta estrutura foram criadas duas tabelas virtuais (views), para organizar e facilitar as consultas ao banco de dados, uma para os registros dos logs (view_paths) e outra para os recursos e atividades (view_instances). Nas tabelas de logs do Moodle são armazenados os registros sobre os acessos a cada módulo no AVA.

As principais inforlmações são: o timestamp (data/hora), o id do curso, o id do usuário, o nome do módulo, o id da instância do módulo acessado. Todas essas informações estão na tabela view chamada view_paths, utilizada para identificar tudo o que o aprendiz acessou. Existem outras como a course_modules, que mantém a 
VII Congresso Brasileiro de Informática na Educação (CBIE 2018)

Anais do XXIX Simpósio Brasileiro de Informática na Educação (SBIE 2018)

identificação única dos recursos/atividades chamada de cmid. Assim, da tabela course_modules, em conjunto com a principal tabela de cada módulo, é possível coletar os dados de todas as instâncias necessárias para a criação do grafo de interação do aluno (trilha de aprendizagem). Para integrar todas as informações relacionadas aos logs e recursos/atividade foi criada a tabela view_instances. Com essa estrutura se facilitou a coleta dos dados no Moodle, pois este possui mais de 250 tabelas e essa simplificação facilitou a geração das TAs baseadas em grafo.

\subsection{Trilhas de Aprendizagem}

Os grafos que foram utilizados nesse trabalho são os idealizados no trabalho de [Ramos et al. 2016], que representam as Trilhas de Aprendizagem (TAs) dos alunos. Segundo [Ramos et al. 2016], na Figura 2 cada vértice representa um recurso/atividade, que por sua vez está associado a uma cor, e o seu diâmetro é proporcional ao seu peso, ponderado pelo número de interações realizadas pelo aluno.

As arestas são de 3 tipos, a padrão (cor verde) representa a estrutura de TA definida pelo professor no AVA, a de avanço (cor azul) representa o percurso que o aluno realizou de um recurso/atividade para outro mais à frente do que a estabelecida pelo professor, e por fim, as arestas de retorno (cor vermelha) representam os retornos realizados pelos alunos a recursos/atividades anteriores. Os valores nas arestas indicam o número de vezes que o aluno percorreu de um vértice $A$ para um vértice $B$ e a seta indica o sentido da trajetória.

Dessas TAs foram extraídos os atributos: média de acesso de vértices, quantidade, dispersão e variância das arestas padrão, avanço e de retorno de cada aluno, estes obtidos por meio dos dados retirados do modelo.

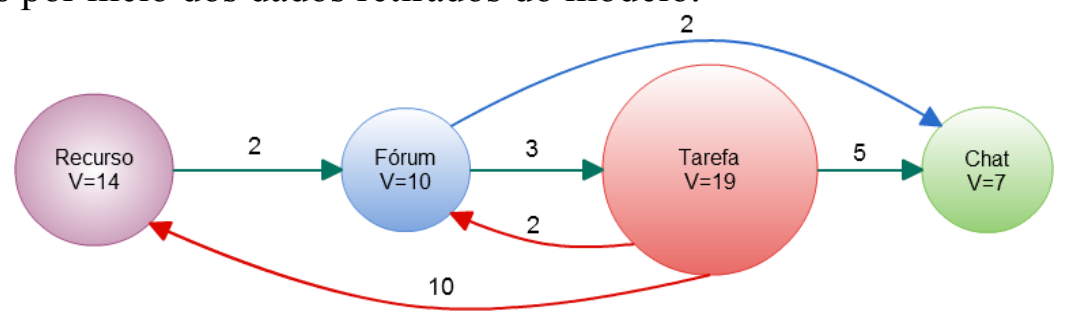

Figura 2: Grafo da Trilha de Aprendizagem. Fonte: [Ramos et al. 2016].

\subsection{Formação de grupos}

Como componente central da estrutura do framework, o plugin M-Cluster é o responsável por gerar os grupos, por meio da alocação dos alunos nos clusters. O MCluster faz a classificação utilizando os atributos extraídos das TAs, a partir do algoritmo K-Means integrado com uma métrica de similaridade.

O K-Means é integrado individualmente com uma métrica de similaridade que pode ser a distância Euclidiana, Manhattan e Cosseno. Cada execução dos dados com uma métrica resulta nos agrupamentos que são exibidos ao professor. O M-Cluster apresenta ao professor os três resultados obtidos com cada métrica.

\subsection{Interface Docente/Tutor}

O instrutor pode selecionar os parâmetros que desejar para a formação dos grupos, como seleção de grupos (nesse caso a palavra grupos refere-se às turmas cadastradas na disciplina ou curso), tipo de exibição, seleção do número de clusteres, seleção dos 
VII Congresso Brasileiro de Informática na Educação (CBIE 2018)

Anais do XXIX Simpósio Brasileiro de Informática na Educação (SBIE 2018)

dados, download de arquivo em formato pdf e representação visual. Para cada parâmetro, o professor pode selecionar a opção desejada, por exemplo, os grupos podem ser visualizados de forma padrão (visão descritiva) ou bolhas (visualização por meio do gráfico de bolhas). Após a definição de todos os parâmetros, a ferramenta é executada e os grupos formados são apresentados na interface docente/tutor.

Como se pode perceber, todos módulos se encaixam para realizar as recomendações de grupos de alunos usando a importância da opinião do professor especialista. Este framework foi baseado na taxonomia de Ounnas, Davis e Millard, (2009). O fluxo ideal de funcionamento, partindo da navegação dos alunos na plataforma e usuários já cadastrados, funciona da seguinte forma: o aluno interage com a plataforma Moodle e, a partir disso, são coletados os logs de arquivos e dados dos alunos. Dessas informações são extraídos os dados para formar as trilhas de aprendizagem dos alunos. Das TAs são extraídos atributos que serão utilizados para classificar e formar os grupos de aprendizes usando o algoritmo K-Means em conjunto com as três métricas de similaridade e os resultados dessas classificações são exibidas ao docente/tutor da turma. Neste passo, a importância da opinião do professor especialista está desde a concepção dos atributos para formar os agrupamentos na fase inicial de concepção do framework, até a escolha dos clusters formados pelo plugin MCluster para serem utilizados nas atividades colaborativas com os alunos. Ao final da classificação, o professor da disciplina faz as avaliações sobre os clusters de alunos que lhes foram recomendados.

O framework proposto por este trabalho é flexível, afinal é possível acrescentar no módulo principal, que correponde ao M-Cluster, mais algoritmos de clusterização e consequentemente, mais métricas de similaridade, podendo pontencializar ainda mais o framework para outras formações de grupos. A ferramenta M-Cluster faz recomendações de grupos de alunos do tipo homogêneo. Com essa flexibilização é possível não só realizar agrupamentos do tipo homogêneo como foi o caso realizado, como também para heterogêneas e híbridas (combinação de homogêneas e heterogêneas). Podemos perceber que a plataforma também é portável, pois sua implementação pode ser reproduzida para outros ambientes virtuais e reutilizável para finalidades relacionadas à recomendação de grupos.

\section{Ferramenta criada a partir do framework}

Na Figura 3, é apresentado o bloco do M-Cluster, a partir dele o professor/tutor tem acesso à interface principal do plugin e desta, pode realizar as configurações que julgar necessárias para obter as sugestões dos agrupamentos dos discentes.

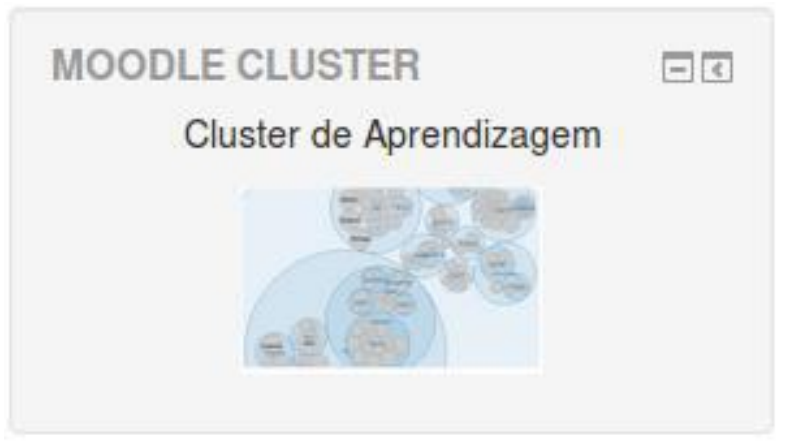

Figura 3: Bloco do M-Cluster 
VII Congresso Brasileiro de Informática na Educação (CBIE 2018)

Anais do XXIX Simpósio Brasileiro de Informática na Educação (SBIE 2018)

A ferramenta, chamada de Moodle Cluster, de [Ramos et al. 2017], gera o modelo de grupos e o armazena em formato JSON. O formato JSON armazena os dados do modelo, que em seguida é renderizado por um script escrito que pode ser usado com o D3.js (biblioteca JavaScript) para criar uma representação visual de grupo e pode também gerar listagem dos grupos para criar a representação descritiva. Na Figura 4, pode-se visualizar um exemplo da representação descritiva. Nesta representação, os grupos recebem uma numeração sequencial, iniciando em zero, em seguida, é extraído da lista de atributos, o nome de cada aluno e apresentado no Moodle. Isso é feito para todos os grupos sugeridos pelo K-Means. Em cada aba, do item Representação visual, ficam os agrupamentos sugeridos de acordo com as métricas utilizadas.

\section{K-Means}

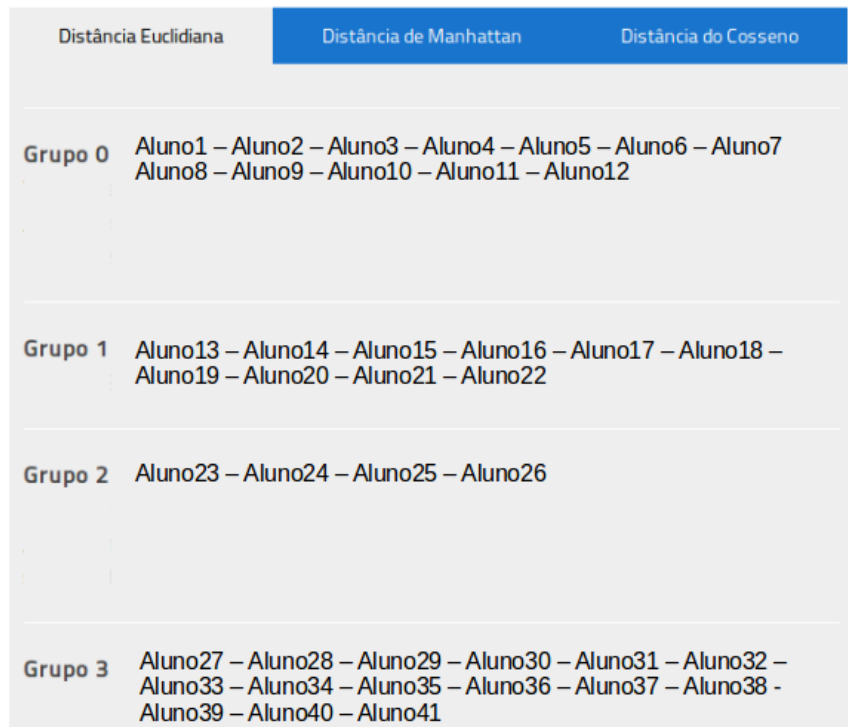

Figura 4: Exemplo de Visualização da Representação Descritiva

A Figura 5 mostra a representação do gráfico de bolhas. Para cada grupo foi criada uma bolha de fundo mais claro, e dentro de cada bolha, foram criadas outras bolhas menores com os nomes dos alunos, com fundo mais escuro.

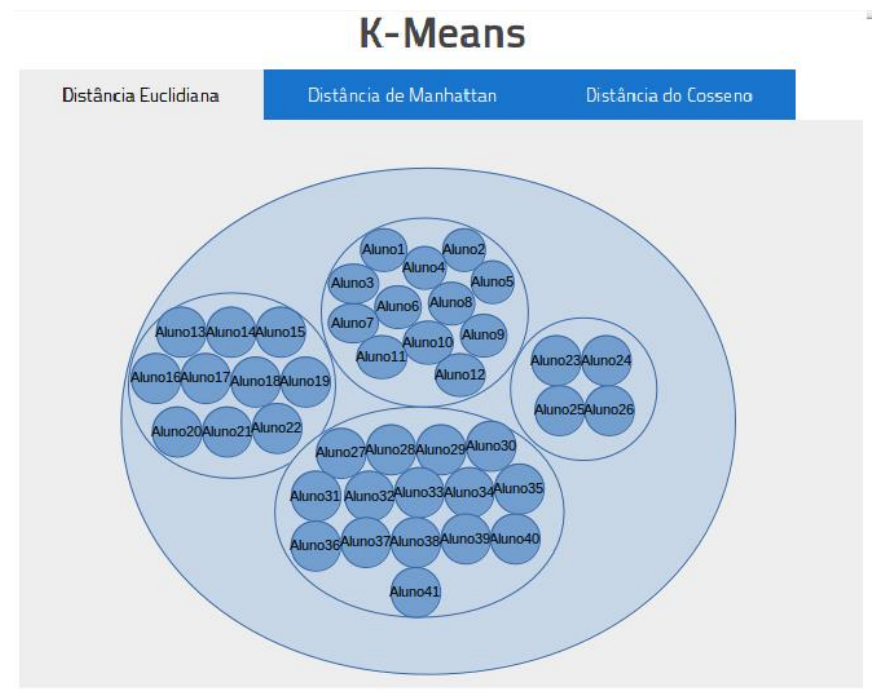

Figura 5: Exemplo de Visualização do Gráfico de Bolhas 
VII Congresso Brasileiro de Informática na Educação (CBIE 2018)

Anais do XXIX Simpósio Brasileiro de Informática na Educação (SBIE 2018)

O Moodle Cluster possui duas representações de sugestões de formação de grupos, uma descritiva e outra visual por meio de um gráfico de bolhas. A ferramenta utiliza o algoritmo K-Means, associado a três métricas de similaridade, juntamente com os atributos obtidos das Trilhas de Aprendizagem, de [Ramos et al. 2016].

\section{Validação Inicial e Resultados}

Foi realizado um estudo de caso em uma turma real em andamento, da disciplina de Matemática Discreta, no semestre de 2016/2, do curso de Ciência da Computação da UFAM, na modalidade presencial e que usava o AVA como suporte às atividades pedagógicas. Participaram do estudo de caso um docente e um tutor (já envolvidos na pesquisa), e 41 alunos matriculados, que assinaram um termo de consentimento livre e esclarecido para participação na pesquisa.

No estudo de caso, o M-Cluster usou os atributos e classificou os alunos com o $K$-Means, obtendo três resultados de agrupamentos, um para cada métrica. Esses grupos sugeridos pela ferramenta foram validados e visualizados pelo docente. Para validar os grupos sugeridos, foram realizadas duas atividades na disciplina. Na primeira atividade, os estudantes escolheram seus parceiros de grupo e na segunda, os grupos foram formados de acordo com as sugestões da ferramenta.

Os experimentos mostraram que, de acordo com o docente especialista, com a ferramenta, $76 \%$ dos alunos igualaram ou superaram as suas notas individuais (essas adquiridas ao formar a dupla para executar as atividades), em relação às notas alcançadas na primeira atividade. Na Figura 6, pode-se visualizar as notas individuais dos alunos analisados. Os alunos desistentes foram excluídos do gráfico.

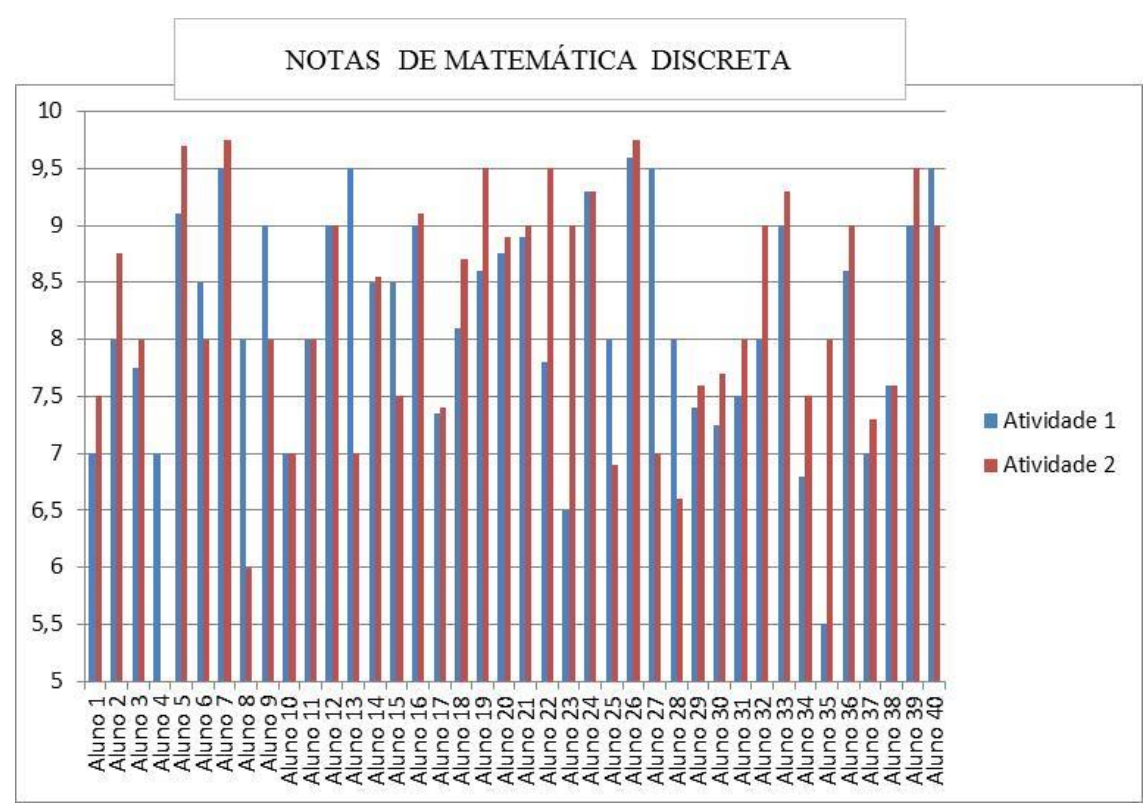

Figura 6: Notas dos alunos de Matemática Discreta.

Foi constatado também, que do total de grupos formados, $30 \%$ eram duplas idênticas às da primeira formação (esses mesmos alunos obtiveram bons resultados nas duas atividades). Esses resultados ainda não são conclusivos, mas já são um ponto de partida para análises futuras mais profundas. 
VII Congresso Brasileiro de Informática na Educação (CBIE 2018)

Anais do XXIX Simpósio Brasileiro de Informática na Educação (SBIE 2018)

\section{Conclusões e Trabalhos Futuros}

Este trabalho apresentou um framework conceitual de recomendação para formação de grupos visando promover a aprendizagem colaborativa no AVA, utilizando a caracterização dos aprendizes. Essa caracterização foi baseada em dados das trilhas de aprendizagem, estas em formato de grafo, para auxiliar o professor no processo de ensino-aprendizagem. Para se chegar ao desenvolvimento do framework, foi realizada uma Revisão Sistemática da Literatura sobre recomendação de grupos para atividades colaborativas nos ambientes virtuais.

A arquitetura geral do framework foi proposta pensando em possíveis inclusões de mais algoritmos de clusterização e métricas de similaridades para possibilitar mais tipos de agrupamentos, como: homogêneos, heterogêneos e híbridos. Assim, o docente poderá escolher qual tipo de agrupamento deseja utilizar para determinada atividade colaborativa. Pode-se citar como contribuições deste trabalho: o agrupamento dos alunos de acordo com as suas trilhas de aprendizagem baseadas no próprio Moodle, as TAs deram subsídios para extrair os atributos mais relevantes para serem usados no algoritmo de clusterização, permitindo gerar as sugestões dos agrupamentos; a identificação de atributos baseados nas TAs em formato de grafo, pois até então não foram observados na literatura sobre recomendação de grupos de alunos em AVAs; a criação de um framework conceitual para detecção automática de grupos de alunos que explora os atributos identificados, servindo de base para pesquisas futuras que queiram aprimorar a investigação sobre recomendação de grupos em AVAs; o desenvolvimento da ferramenta M-Cluster no formato de plugin para o Moodle contribui como um exemplo prático da aplicação do framework, podendo o plugin ser integrado em qualquer AVA.

As possibilidades de trabalhos futuros são inúmeras. Dentre os de maior relevância, podemos citar: (i) criação da opção de formar tanto grupos homogêneos quanto heterogêneos, dando ao docente a possibilidade de agrupar indivíduos que tenham conhecimentos complementares; (ii) integração da ferramenta com as técnicas de aprendizagem colaborativa, pois possibilitará formar os grupos de acordo com a técnica selecionada pelo professor; (iii) associação de forma automática do resultado do grupo gerado pela ferramenta com a atividade postada no módulo do curso, de forma que seja possível a disponibilização automática dos grupos como anexo na descrição da atividade.

\section{Agradecimentos}

Parte dos resultados apresentados neste artigo foi obtida através de atividades de P\&D do projeto PROMOBILE patrocinado pela Samsung nos termos da lei federal brasileira $n^{\circ} 8.248 / 91$. Agradecemos à FAPEAM pelo suporte financeiro concedido para a realização do projeto e ao Programa de Apoio a Jovens Doutores (PJD/UFAM), Edital PROPESP/UFAM 041/2016.

\section{Referências}

Abnar, S., Orooji, F. e Taghiyareh, F. An evolutionary algorithm for forming mixed groups of learners in web based collaborative learning environments. In: Proceedings IEEE International Conference on Technology Enhanced Education, ICTEE, 2012. 
VII Congresso Brasileiro de Informática na Educação (CBIE 2018)

Anais do XXIX Simpósio Brasileiro de Informática na Educação (SBIE 2018)

Barkley, E. F., Major, C. H. e Cross, K. P. (2014) "Collaborative Learning Techniques", 2 ed, San Francisco, Jossey-Bass.

Bezdek, J. C. (1981) "Pattern Recognition with Fuzzy Objective Function Algorithms", Plenum Press, New York.

Jagadish, D. (2014) "Grouping in collaborative e-learning environment based on interaction among students", In: IEEE International Conference on Recent Trends in Information Technology.

Le, T. V. (1995) "Evolutionary fuzzy clustering", In: IEEE International Conference on Evolutionary Computation, pp. 753-758.

MacQueen, J. (1967) "Some methods for classification and analysis of multivariate observations", In: Proceedings of the Fifth Berkeley Symposium on Mathematical Statistics and Probabilities, 1, pp. 281-296.

Montazer, G. A. e Rezaei, M. S. (2012) "A new approach in e-learners grouping using Hybrid Clustering Method", In: International Conference on Education and eLearning Innovations, p. 1-5.

Natalli, L. E e Menezes, S. C. (2012) "FrameColab: Um framework para criação de Ambientes Colaborativos", In: XXIII Simpósio Brasileiro de Informática na Educação.

Ramos, D. B., Oliveira, E. H. T., Ramos, I. M. M., e Oliveira, K. M. (2015) "Trilhas de Aprendizagem em Ambientes Virtuais de Ensino-aprendizagem: Uma Revisão Sistemática da Literatura", In: XXVI Simpósio Brasileiro de Informática na Educação, 26, pp. 338-347. Maceió.

Ramos, I. M. M., Ramos, D. B., Nascimento, P. B., Amaral, G. S., Gadelha, B. F. e Oliveira, E. H. T. (2017) "M-Cluster: Uma ferramenta de Recomendação para Formação de Grupos em Ambientes Virtuais de Aprendizagem", In: Simpósio Brasileiro de Informático de Educação, p. 1657-1666.

Ramos, I. M. M, Ramos, D. B., Nascimento, P. B., Gadelha, B. F., e Oliveira, E. H. T. (2016) Formação de Grupos em Ambientes Virtuais de Aprendizagem: Uma Revisão Sistemática da Literatura. In: XXVII Simpósio Brasileiro de Informática na Educação, 26, p. 310-319.

Santos, L. N., Castro, A. N. e Castro, T. H. C. (2007) "Alteração no Modelo de Grupos do Moodle para Apoiar a Colaboração", In: XVIII Simpósio Brasileiro de Informática na Educação, p. 24-35.

Yathongchai, C., Angskun, T., Yathongchai, W. e Angskun, J. (2013) "Leaner Classification Based on Learning Behavior and Performance", In: IEEE Conference on Open Systems, p. 66-70. 